\title{
Evaluation of Feature Selection Measures for Steganalysis
}

\author{
G.K. Rajput and R.K. Agrawal \\ School of Computer and Systems Sciences, \\ Jawaharlal Nehru University, New Delhi-110067 \\ gauravrajput1@gmail.com, rka@mail.jnu.ac.in
}

\begin{abstract}
Steganalysis has attracted researchers attention overwhelmingly in last few years which discriminate stego images from non-stego images. The performance of a Steganalysis depends not only on the choice of classifier but also on features that are used to represent the image. Features extracted from images may contain irrelevant and redundant features which makes them inefficient for machine learning. Relevant features not only decrease the processing time to train a classifier but also provide better generalization. In this paper, kullback divergence measure, chernoff distance measure and linear regression are used for relevant feature selection. The performance of steganalysis using different measures used for feature selection is compared and evaluated in terms of classification error and computation time of training classifier. Experimental results show that Linear regression measure used for feature selection outperforms other measures used for feature selection in terms of both classification error and compilation time.
\end{abstract}

Keywords: Steganalysis, Feature Selection, Linear Regression, Kullback Divergence, Chernoff Distance Measure.

\section{Introduction}

Information hiding techniques have gained enormous popularity among researchers due to increasing threats for security, especially after 9/11 incident. Research community is involved in study of methods of secure communication as well as methods of detection of the covert communication between the two parties. Steganography is commonly used technique for data hiding in carrier signals. The carrier signal undergoes modification once the data to be sent is embedded using some technique. Images are often used as a carrier because of their extensive availability with high resolution of pixels. In recent years, detecting the presence of hidden messages has posed significant challenges to research community [2, 3, 4, 6, 10, 17]. Steganalysis is art of discriminating such suspicious signals from a large number of inoffensive signals over a communication channel. Several techniques for steganalysis were proposed in literature [4,11,18]. Since the data hiding may involve different image formats, different embedding algorithms, and various steganographic keys, steganalysis turns out to be more difficult and challenging task. Recently, Farid [2, 3] used wavelet-like decomposition to build higher-order statistical models of natural images in terms of mean, variance, skewness, and kurtosis of coefficients of wavelet subbands as features and employed classifier to discriminate between stego and non-stego images. However, the resultant features may contain noisy,

S. Chaudhury et al. (Eds.): PReMI 2009, LNCS 5909, pp. 432-439. 2009.

(C) Springer-Verlag Berlin Heidelberg 2009 
irrelevant or redundant features which makes them inefficient for machine learning. In fact, the presence of irrelevant and redundant features may deteriorate the performance of the classifier and requires high computation time and other resources for training and testing the data. Hence, in order to enhance the performance of stegoanalysis in terms of accuracy and time required to detect, there is need to identify a set of relevant features.

Feature selection is used to remove such noisy, irrelevant, and redundant features. There are two major approaches to feature selection: filter and wrapper approach [5] 7, 8]. Most filter methods employ statistical characteristics of data for feature selection which requires less computation. It independently measures the importance of features without involving any classifier. Since, the filter approach does not take into account the learning bias introduced by the final learning algorithm, it may not be able to select the most relevant set of features for the learning algorithm. On the other hand, wrapper methods tend to find features better suited to the predetermined learning algorithm resulting in better performance. But, it tends to be computationally more expensive since the classifier must be trained for each candidate subset.

Feature ranking approaches have been widely investigated for feature selection [12 14, 15] in literature. Since in most of feature ranking approaches features are evaluated using statistical characteristics of the data, different feature ranking methods measure different characteristics of data. Therefore, the informative features selected by different ranking methods may be different. Another disadvantage associated with feature ranking methods is that they ignore the correlation among the features because of their univariate approach. Hence the selected features subset may have low discriminatory capacity and increased redundancy. In literature to remove redundancy a forward/backward feature selection method or its combinations are used with a measure that selects relevant and non redundant features. Among the most widely used filter methods [6] for feature selection, there are techniques based on statistical separability measures which allow one to select a suitable subset of features by assigning the degree of interclass separability associated with each subset considered. In particular, Kullback Divergence, Chernoff distance measures and linear regression are commonly employed by research community. In this paper, we compare and evaluate these measures to determine relevant features for steganlaysis.

Our work is organized as follows: Feature extraction using higher order statistical model is included in section 2. A brief introduction of separability measures employed for features selection techniques are discussed in section 3. Experimental results on a database of natural images are shown in section 4 and section 5 contains conclusions.

\section{Feature Extraction Using Higher Order Image Statistics}

Although the presence of embedded message is most often not detectable to the human eye, but it may nevertheless changes the statistics of an image. The distortions in the resulting stegoimages can be analyzed by comparing the statistical properties of both cover and stegoimages [18, 19]. In literature, techniques [19,20] are available to detect such changes based on first order statistical distributions of intensity or transform coefficients. The disadvantage of this analysis is that simple counter-measures that match first order statistics are likely to thwart detection. Farid [2] has pointed out that steganalysis based on higher-order statistical models may detect stegoimages. It has been 
observed across a large number of natural images that there exist strong higher-order statistical regularities within a wavelet-like decomposition. The embedding of a message may significantly change the statistics of image and thus becomes detectable.

The decomposition of image is possible by using separable quadrature mirror filters (QMFs). Thus, the frequency space is divided into multiple scales and orientations. This can be accomplished by applying separable lowpass and highpass filters along the image axes generating a vertical, horizontal, diagonal, and lowpass subband. The diagonal, horizontal, and vertical subband at scale $i=1,2, \ldots, n$ are represented as $D_{i}(x, y)$, $H_{i}(x, y)$, and $V_{i}(x, y)$ respectively. Subsequent scales are obtained by recursively filtering the lowpass subband. Farid [2,3] pointed out that using above decomposition the statistical model containing the mean, variance, skewness and kurtosis of the subband coefficients for each orientation and scales can be obtained for $i=1$ to $n$. This characterizes the basic coefficient distributions statistically. The second set of statistics is based on the errors in an optimal linear predictor of coefficient magnitude. It is pointed out [2,3] that the subband coefficients of the image are correlated to their spatial, orientation and scale neighbors. Taking this into account, $V_{i}(x, y)$, a vertical band at scale $i$, can be represented in terms of neighboring pixels in spatial domain as:

$$
\begin{array}{r}
V_{i}(x, y)=w_{1} V_{i}(x-1, y)+w_{2} V_{i}(x+1, y)+w_{3} V_{i}(x, y-1)+w_{4} V_{i}(x, y+1) \\
+w_{5} V_{i+1}(x / 2, y / 2)+w_{6} D_{i}(x, y)+w_{7} D_{i+1}(x / 2, y / 2)
\end{array}
$$

Where $w_{k}$ denotes scalar weighting values. In more compact form, it can be expressed as

$$
\boldsymbol{V}=Q \boldsymbol{w},
$$

Where $\boldsymbol{w}=\left(w_{1}, w_{2}, \ldots \ldots \ldots, w_{7}\right)^{T}$, the vector $\boldsymbol{V}$ contains the coefficient magnitudes of $V_{i}(x, y)$ strung into a column vector and the columns of the matrix $\mathrm{Q}$ contain the neighboring coefficient magnitudes as specified in (1) also strung out in column vectors. To determine coefficients of quadratic error function is defined [2,3] as

$$
E(\boldsymbol{w})=[\boldsymbol{V}-Q \boldsymbol{w}]^{2}
$$

This error function $E(\boldsymbol{w})$ can be minimized by differentiating equation (3) and substituting it equal to zero yields:

$$
\boldsymbol{w}=\left(Q^{T} Q\right)^{-1} Q^{T} \boldsymbol{V}
$$

From the above, order statistics such as the mean, variance, skewness, and kurtosis can be evaluated. Similarly, the above procedure can be repeated to get the subbands $H_{i}(x, y), D_{i}(x, y)$. Since, there are four statistics and linear predictor for three subband are computed for $(n-1)$ levels, we have total $12(n-1)$ error statistics and $12(n-1)$ coefficient statistics. Thus, these $24(n-1)$ statistics altogether will form a feature vector of the image. 


\section{Forward Feature Selection Techniques}

Feature ranking is commonly used to determine a subset of relevant features. However, the disadvantage of feature ranking method is that they ignore the correlations between features. Hence the features selected may contain redundant information. Some of the methods suggested in literature for removing redundancy are Chernoff distance measure, Kullback divergence measure [14] and linear regression [16].

In order to obtain a quantitative measure of how separable are two classes, a distance measure can be easily extracted from some parameters of the data. A very important aspect of probabilistic distance measures is that a number of these criteria can be analytically simplified in the case when the class conditional p.d.f.s $p\left(X_{i} \mid C_{i}\right)$ follows multivariate normal distribution. In literature, for multivariate normal distribution for two classes, KD and CD measures are given as follows [14]:

$$
\begin{aligned}
& J_{k}^{d}=\frac{1}{2}\left(\mu_{k}^{2}-\mu_{k}^{1}\right)^{T}\left(\left(\Sigma_{k}^{1}\right)^{-1}+\left(\Sigma_{k}^{2}\right)^{-1}\right)\left(\mu_{k}^{2}-\mu_{k}^{1}\right)+\frac{1}{2} \operatorname{tr}\left(\left(\Sigma_{k}^{1}\right)^{-1} \Sigma_{k}^{2}+\left(\Sigma_{k}^{2}\right)^{-1} \Sigma_{k}^{1}-2 I_{k}\right) \\
& J_{k}^{c}=\frac{1}{2} \beta(1-\beta)\left(\mu_{k}^{2}-\mu_{k}^{1}\right)^{T}\left[(1-\beta) \Sigma_{k}^{1}+\beta \Sigma_{k}^{2}\right]^{-1}\left(\mu_{k}^{2}-\mu_{k}^{1}\right)+\frac{1}{2} \log \frac{\left|(1-\beta) \Sigma_{k}^{1}+\beta \Sigma_{k}^{2}\right|}{\left|\Sigma_{k}^{1}\right|^{1-\beta}\left|\Sigma_{k}^{2}\right|^{\beta}}
\end{aligned}
$$

where $\mu_{k}^{i}$ is a mean vector and $\Sigma_{k}^{i}$ is a covariance matrix of k-dimensional data for class $C_{i}, i=1,2$. The regression analysis considers the relations between the selected features which minimizes redundancy. While using regression analysis for data a multiple regression model is considered because there can be many features which could affect the presence or absence of stegoimage. A multiple regression model with a target variable $y$ and multiple variables $X$ is given by [16]:

$$
y=\beta_{0}+\beta_{1} X_{1 i}+\beta_{2} X_{2 i}+\cdots+\beta_{n} X_{n i}+\xi_{i}, \quad i=1,2, \ldots, n
$$

Where $\beta_{0}, \beta_{1}, \ldots, \beta_{n}$ are constants estimated by observed values of $X$ and class label $y$ and is estimated by normal distribution having mean zero and a variance $\sigma^{2}$.

The error sum of squares SSE which is sum of the squared residuals is given by

$$
S S E=\sum_{n=0}^{n}\left(y_{i}-\text { predicted } y_{i}\right)
$$

A large value of SSE means that the regression is predicted poorly. The total sum of squares is given by

$$
S S T O=\sum_{n=0}^{n}\left(y_{i}-\bar{y}\right)
$$

Where $\bar{y}$ is the average of $y_{i}$. In a regression model the choice of features which best explains the class label depends on the value of $\mathcal{R}^{2}$ given by

$$
\mathcal{R}^{2}=1-\frac{S S E}{S S T O}
$$




\section{Experimental Setup and Results}

We prepared a database of 1500 natural images taken from different sources i.e www.1000pictures.com, www.1000wallpapers.com All the images were in JPEG format. The image resolutions were ranging from $800 \times 600$ to $1600 \times 1200$. We first resized each one of these images to $640 \times 480$ pixels images and embedded message images of six different resolutions $256 \times 256,128 \times 128,64 \times 64,32 \times 32,16 \times 16$, and $8 \times 8$ into cover image using OUTGUESS [19].

We have created 1000 non-stego images and 1000 stego images. Features are extracted from each one of the grey images using Haar wavelet. Each image is represented in terms of 72 statistics for four level wavelet decomposition. To remove redundancy from the selected pool of features three methods are compared: kullback divergence measure, chernoff distance measure and linear regression. For chernoff distance features are selected using 3 different values ranging from 0.1 to 0.9 with an increment of 0.4. We have used the following classifiers to evaluate the performance of the feature selection methods: naive Bayes Classifier (naivebc), Logistics linear classifier (loglc), Fisher linear classifier (fisherc), Nearest mean classifier (nmc), Normal Density based classifier-Independent features (udc), Normal Density based linear classifier (ldc), Normal Density based quadratic classifier (qdc). Classification error is computed using ten cross-validation. All the simulations are done using matlab. Tables 1-3 show the minimum classification error achieved with different classifiers along with the number of features for different measures. For chernoff distance measure the minimum classification error achieved for optimal value of $\beta$ is shown in Tables 1-3. The best results in each category are indicated in bold. We observe the following from Tables 1-3:

1. The minimum classification error is achieved with linear regression for all classifiers and for different size of embedding.

2. The number of features required to obtain minimum classification error is significantly smaller using linear regression in comparison to baseline, kullback divergence measure and chernoff distance measure using all classifiers and different size of embedding.

3. The performances of ldc, fisherc and loglc are comparable and better than other classifiers in terms of classification error for all sizes of embedding used in experiments.

Table 1. Comparative results of classification error and minimum number of features for Size $8 \times 8$ and $16 \times 16$

\begin{tabular}{|l|l|l|l|l|l|l|l|l|l|l|l|l|l|l|}
\hline & \multicolumn{9}{|c|}{ Size $=8 \times 8$} & \multicolumn{4}{c|}{ Size $=16 \times 16$} \\
\hline & Baseline & \multicolumn{2}{|c|}{ JD } & \multicolumn{2}{|c|}{ JC } & \multicolumn{2}{|c|}{ Linear } & \multicolumn{1}{c|}{ Baseline } & \multicolumn{1}{|c|}{ JD } & \multicolumn{3}{c|}{ JC } & \multicolumn{2}{c|}{ Linear } \\
\hline & ErrAll & fea & ErrJD & beta & ErrJC & fea & ErrLin & ErrAll & fea & ErrJD & beta & ErrJC & fea & ErrLin \\
\hline Ldc & 0.3170 & 67 & 0.3045 & $38, .9$ & 0.2985 & 23 & $\mathbf{0 . 2 9 0 5}$ & 0.3210 & 39 & 0.3040 & $38, .5$ & 0.2985 & 24 & $\mathbf{0 . 2 6 8 0}$ \\
\hline Fisherc & 0.3240 & 67 & 0.3045 & $46, .9$ & 0.3000 & 24 & $\mathbf{0 . 2 9 0 5}$ & 0.3160 & 34 & 0.3045 & $38, .5$ & 0.2955 & 20 & $\mathbf{0 . 2 9 1 0}$ \\
\hline Naivebc & 0.5605 & 64 & 0.4705 & $16, .9$ & 0.4430 & 2 & $\mathbf{0 . 4 3 6 0}$ & 0.5390 & 27 & 0.4660 & $21, .9$ & 0.4350 & 4 & $\mathbf{0 . 3 8 6 0}$ \\
\hline Udc & 0.4900 & 65 & 0.4860 & $22, .5$ & 0.4875 & 9 & $\mathbf{0 . 4 2 6 0}$ & 0.5025 & 55 & 0.4945 & $37, .9$ & 0.4930 & 9 & $\mathbf{0 . 3 8 5 5}$ \\
\hline Qdc & 0.5000 & 37 & 0.4735 & $35, .5$ & 0.4670 & 7 & $\mathbf{0 . 3 4 4 5}$ & 0.5090 & 31 & 0.4760 & $24, .5$ & 0.4675 & 7 & $\mathbf{0 . 3 0 8 5}$ \\
\hline Nmc & 0.4990 & 47 & 0.4970 & $52, .1$ & 0.4950 & 4 & $\mathbf{0 . 4 1 6 5}$ & 0.5020 & 3 & 0.4975 & $1, .1$ & 0.4975 & 1 & $\mathbf{0 . 4 0 9 0}$ \\
\hline Loglc & 0.3090 & 63 & 0.3005 & $44, .1$ & 0.2960 & 24 & $\mathbf{0 . 2 9 3 5}$ & 0.3065 & 34 & 0.2995 & $44, .1$ & 0.2985 & 18 & $\mathbf{0 . 2 6 7 0}$ \\
\hline
\end{tabular}


Table 2. Comparative results of classification error and minimum number of features for sizes $32 \times 32$ and $64 \times 64$

\begin{tabular}{|l|l|l|l|l|l|l|l|l|l|l|l|l|l|l|l|}
\hline & \multicolumn{9}{|c|}{ Size $=32 \times 32$} & \multicolumn{4}{c|}{ Size $=64 \times 64$} \\
\hline & Baseline & \multicolumn{2}{|c|}{ JD } & \multicolumn{2}{|c|}{ JC } & \multicolumn{2}{|c|}{ Linear } & \multicolumn{1}{|c|}{ Baseline } & \multicolumn{3}{|l|}{ JD } & \multicolumn{3}{|c|}{ JC } & \multicolumn{2}{c|}{ Linear } \\
\hline & ErrAll & fea & ErrJD & beta & ErrJC & fea & ErrLin & ErrAll & fea & ErrJD & beta & ErrJC & fea & ErrLin \\
\hline Ldc & 0.3155 & 54 & 0.3075 & $44, .1$ & 0.3005 & 23 & $\mathbf{0 . 2 9 2 0}$ & 0.3095 & 60 & 0.3090 & $43, .1$ & 0.2990 & 37 & $\mathbf{0 . 2 9 2 5}$ \\
\hline Fisherc & 0.3210 & 51 & 0.3085 & $36, .9$ & 0.3015 & 21 & $\mathbf{0 . 2 9 3 0}$ & 0.3260 & 25 & 0.3130 & $37, .1$ & 0.3020 & 19 & $\mathbf{0 . 2 9 2 5}$ \\
\hline Naivebc & 0.5385 & 19 & 0.4675 & $13, .9$ & 0.4375 & 2 & $\mathbf{0 . 4 2 7 5}$ & 0.5325 & 6 & 0.4725 & $15, .5$ & 0.4420 & 2 & $\mathbf{0 . 4 3 3 0}$ \\
\hline Udc & 0.4985 & 38 & 0.4865 & 29,5 & 0.4850 & 9 & $\mathbf{0 . 4 2 3 0}$ & 0.5035 & 43 & 0.4925 & $47, .9$ & 0.4895 & 11 & $\mathbf{0 . 4 2 2 5}$ \\
\hline Qdc & 0.5035 & 23 & 0.4650 & $22, .1$ & 0.4595 & 2 & $\mathbf{0 . 3 4 0 0}$ & 0.5025 & 26 & 0.4705 & $17, .5$ & 0.4620 & 11 & $\mathbf{0 . 3 4 0 5}$ \\
\hline Nmc & 0.5030 & 72 & 0.4910 & $5, .5$ & 0.4925 & 4 & $\mathbf{0 . 4 2 6 0}$ & 0.5015 & 46 & 0.4960 & $2, .1$ & 0.4940 & 4 & $\mathbf{0 . 4 1 3 5}$ \\
\hline Loglc & 0.3025 & 60 & 0.2965 & $66, .5$ & 0.2975 & 17 & $\mathbf{0 . 2 9 2 5}$ & 0.3135 & 66 & 0.3010 & $39, .9$ & 0.2965 & 25 & $\mathbf{0 . 2 9 2 0}$ \\
\hline
\end{tabular}

Table 3. Comparative results of classification error and minimum number of features for Size $128 \times 128$ and $256 \times 256$

\begin{tabular}{|c|c|c|c|c|c|c|c|c|c|c|c|c|c|c|}
\hline & \multicolumn{7}{|c|}{ Size $=128 \times 128$} & \multicolumn{7}{|c|}{ Size $=256 \times 256$} \\
\hline & Baseline & & JD & & $\mathrm{C}$ & & inear & Baseline & & JD & & $\bar{C}$ & & inear \\
\hline & ErrAll & fea & ErrJD & beta & ErrJC & fea & ErrLin & ErrAll & fea & ErrJD & beta & ErrJC & fea & ErrLin \\
\hline $\mathrm{Ldc}$ & 0.3195 & 64 & 0.3170 & $42, .1$ & 0.3015 & 27 & 0.2925 & 0.2900 & 67 & 0.2820 & $55, .9$ & 0.2675 & 24 & 0.2680 \\
\hline Fisherc & 0.3355 & 69 & 0.3130 & $45, .5$ & 0.3075 & 22 & 0.2960 & 0.3360 & 67 & 0.2770 & 46,9 & 0.2730 & 20 & 0.2965 \\
\hline Naivebc & 0.5085 & 25 & 0.4900 & $29, .5$ & 0.4430 & 2 & 0.4360 & 0.4360 & 64 & 0.4330 & $22, .5$ & 0.4125 & 4 & 0.3860 \\
\hline Udc & 0.4955 & 69 & 0.4915 & $35, .5$ & 0.4910 & 11 & 0.4170 & 0.4780 & 65 & 0.4765 & $71, .5$ & 0.4710 & 9 & 0.3855 \\
\hline Qdc & 0.5015 & 56 & 0.4875 & $34, .1$ & 0.4730 & 12 & 0.3415 & 0.4745 & 37 & 0.4605 & $25, .5$ & 0.4555 & 7 & 0.3085 \\
\hline $\mathrm{Nmc}$ & 0.4985 & 22 & 0.4945 & $25, .5$ & 0.4950 & 2 & 0.3930 & 0.4970 & 47 & 0.4865 & $45, .5$ & 0.4930 & 1 & 0.4090 \\
\hline Loglc & 0.3190 & 70 & 0.3190 & $41, .1$ & 0.3020 & 22 & 0.2905 & 0.2835 & 63 & 0.2795 & $43, .5$ & 0.2695 & 18 & 0.2670 \\
\hline
\end{tabular}

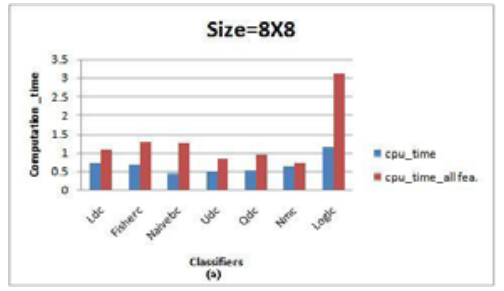

Fig. 1. Variation of Computation time (a) $8 \times 8 \quad$ (b) $128 \times 128$

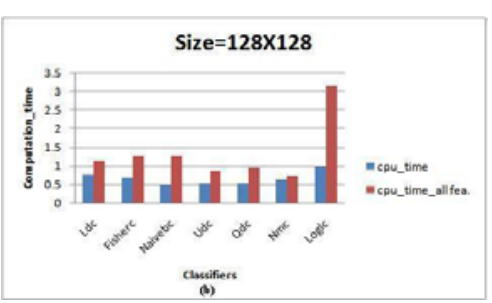

The training time of different classifiers using features selected to achieve minimum classification error using linear regression measure and using baseline (all features) for two $(8 \times 8$ and $128 \times 128)$ sizes of embedding are shown in Figure 1 . It can be observed from Figure 1 that the training time required using features selected by linear regression measure is very small in comparison to training time of classifier using baseline (all features) for all sizes of embedding used in experiments. The time difference observed is more in case of $\log c$ classifier.

\section{Conclusions}

The performance of a classifier depends on the choice of features and classifier for any pattern recognition system. Features based on higher order statistics are extracted from 
stego and non-stego images using wavelet decompositions. Feature extracted from image may contain irrelevant and redundant features which makes them inefficient for machine learning. Hence, relevant features which provide minimum classification error are selected using kullback divergence measure, chernoff distance measure and linear regression. The performance of steganalysis using different measures used for feature selection is compared and evaluated in terms of classification error and computation time of training classifier. Experimental results show that classification error with features selected using linear regression is less in comparison to other measures used for feature selection. Also the relevant features selected using linear regression is much smaller in size in comparison to other measures with all classifiers. Hence linear regression measure used for feature selection outperforms other measures in terms of training computation time of a classifier. To improve the performance of the Steganalysis, future work could include the following: (i) identifying the type of Steganography algorithm used for embedding the secret message, (ii) To locate, retrieve and analyze the embedded message to infer the information hidden in image.

\section{References}

1. Harmsen, J., Pearlman, W.: Steganalysis of additive noise modelable information hiding. In: Proc. SPIE Security and Watermarking of Multimedia Contents V, Santa Clara, CA, January 2003, vol. 5022 (2003)

2. Farid, H.: Detecting hidden messages using higher-order statistical models. In: Proc. of the IEEE int'l Conf. on Image processing 2002, vol. 2, pp. 905-908. IEEE, New York (2002)

3. Lyu, S., Farid, H.: Steganalysis using higher-order image statistics. IEEE Trans. Inf. Forensics Security 1(1), 111-119 (2006)

4. Mehrabi, M.A., Faez, K., Bayesteh, A.R.: Image Steganalysis on Statistical moments of Wavelet Subband histograms in Different Frequencies and Support Vector Machine. In: Proc. of Third int'l Conf. on Natural Computation, August 24-27 (2007)

5. Guyon, I., Elisseeff, A.: An Introduction to Variable and feature Selection. Journal of Machine Learning Research (3), 1157-1182 (2003)

6. Lie, W.-n., Lin, G.-S.: A Feature-Based Classification Technique for Blind Image Steganalysis. Proc. IEEE Transaction on Multimedia 7(6) (December 2005)

7. Kohavi, R., John, G.: Wrapper for feature subset selection. Artificial Intelligence (1-2), $273-$ 324 (1997)

8. Ruiz, R., et al.: Incremental wrapper based gene selection from microarray data for cancer classification. Pattern Recognition 39(12), 2383-2392 (2006)

9. Fridrich, J., Goljan, m.: Practical steganalysis: State of the art. In: SPIE photonics West, Electronic Imaging, San Jose, CA (2002)

10. Johanson, N., Jajodia, S.: Exploring steganography: seeing the unseen. IEEE Computer, 2634 (1998)

11. Westfeld, A., Pfitzmann, A.: Attacks on steganographic systems. In: Pfitzmann, A. (ed.) IH 1999. LNCS, vol. 1768, pp. 61-75. Springer, Heidelberg (2000)

12. Guyon, I., et al.: Gene Selection for cancer classification using support vector machine. Machine Learning (46), 263-268 (2003)

13. Duda Richard, O., Hart Peter, E., Stork David, G.: Pattern Classification, 2nd edn. Wiley India (P) Ltd., Chichester 
14. Pierre, A.D., Kittler, J.: Pattern Recognition: A Statistical Approach. PHI (1982)

15. Tibsrani, R., et al.: Diagnosis of multiple cancer types by shrunken centriods of gene expression. Proc. Natl. Acad. Sci. USA (99), 6567-6572 (2002)

16. Han-Saem, P., et al.: Forward selection Method with regression analysis for optimal gene selection in cancer classification. International Journal of Computer Mathematics 84(5), 653668 (2007)

17. Kahn, D.: The history of steganography. In: Anderson, R. (ed.) IH 1996. LNCS, vol. 1174. Springer, Heidelberg (1996)

18. Pevný, T., Fridrich, J.: Determining stego algorithm for JPEG images. IEEE Proc. -Iinf. Security 153(3) (September 2006)

19. Provos, N., Honeyman, P.: Hide and Seek: An Introduction to Steganography. In: IEEE Security and Privacy. The IEEE Computer Society, Los Alamitos (2003)

20. Wang, Y.: Optimized Feature Extraction for Learning-Based Image Steganalysis. IEEE Transactions on Information Forensics and Security @(1) (March2007) 